\title{
ASSOCIATION OF VITAMIN D DEFICIENCY WITH CARPAL TUNNEL SYNDROME
}

\author{
Hasanain A Al-Khalidi' ${ }^{1}$, Ahmed Muhi Fahad ${ }^{2}$ \\ ${ }^{1}$ Faculty of Medicine, Kufa University; Middle Euphrates Neurosciences Center - Najaf, Iraq \\ ${ }^{2}$ Department of Cardiovascular Surgery, Najaf Health Directorate, Al-Sadder Teaching Medical City - Najaf, Iraq
}

\begin{abstract}
Vitamin D deficiency is defined when serum $25(\mathrm{OH}) \mathrm{D}$ concentration is less than $25 \mathrm{ng} / \mathrm{ml}$; it is associated with many painful syndromes, such as carpal tunnel syndrome (CTS). CTS is a common entrapment neuropathy that is caused by compression of the median nerve at the wrist joint. We sought to describe the relationship between CTS and vitamin D deficiency, as well as the effect of vitamin D therapy on decreasing the severity of CTS-associated pain. We selected 32 out of 44 patients who presented with CTS at our hospital in Najaf province, Iraq. We then clinically assessed the severity of pain using the visual analog score (VAS) and an electromyography/nerve conduction study (EMG/NCS). We tested all patients for serum vitamin $D$ levels before and after 3 months of vitamin D therapy. CTS was most common in females aged 40 to 49 years who were righthand dominant. There was a significant relationship between vitamin $D$ deficiency and pain score before and after treatment with vitamin $D$. The increased level of vitamin $D$, the lower the pain score. Thus, there is a clear link between vitamin $D$ therapy and improvement of clinical symptoms in patients with CTS.
\end{abstract}

Key words: vitamin D deficiency, carpal tunnel syndrome, neuropathy, VAS score

\section{INTRODUCTION}

Vitamin $D$ is a steroid molecule that is derived from two sources, mainly from 7-dehydrocholesterol via the effect of ultraviolet light on the skin but also from the diet [1]. The main metabolite of vitamin $D$ is 25 hydroxyvitamin $\mathrm{D}(25(\mathrm{OH}) \mathrm{D})$, which has a halflife of 15 to 20 days in the body [2]. Vitamin D plays a role in musculoskeletal health and its deficiency is associated with gastrointestinal, cardiovascular, and neuropsychiatric disorders, and cancer [2, 3]. It is also linked to many painful syndromes, as it has an effect on nociceptive and inflammatory pain [4]. Vitamin D deficiency is defined when serum $25(\mathrm{OH})$ $D$ is less than or equal to $25 \mathrm{ng} / \mathrm{ml}(50 \mathrm{mmol} / \mathrm{l})$ according to Forrest and Stuhldreher in the US [5], while according to a consensus adopted in 2019 by the Bulgarian Society of Endocrinology, the Bulgarian Society of Rheumatology, the Bulgarian Pediatric Association, and the Bulgarian Society of Clinical Laboratory, the standards for vitamin D in Bulgaria are as follows: Severe deficiency $<25.00 \mathrm{nmol} / /$; insufficiency $25-49.99 \mathrm{nmol} / /$; and sufficiency $\geq 50.00$ $\mathrm{nmol} / /$ [6]. In Iraq, the prevalence of vitamin D deficiency is around $50 \%$, especially among females of reproductive age [7].

Carpal tunnel syndrome (CTS) is a common entrapment neuropathy, which is caused by compression of the median nerve at the wrist joint, and it accounts for about $90 \%$ of nerves affected $[1,8,9]$. Etiological factors include diabetes mellitus, obesity, hyperlipidemia, rheumatoid arthritis, hypothyroidism, recurrent twisting movements, and pregnancy; however, it is usually idiopathic $[1,2,9]$. The diagnosis of CTS is dependent on clinical presentations plus clinical tests, such as Phalen's test or Tinel's test $[10,11]$. The diagnostic modality of choice for CTS is electromyography/nerve conduction study (EMG/ NCS), which can be used to assess the severity of CTS [10]. The severity of pain can be clinically assessed using various scoring systems, including the Boston carpal tunnel questionnaire (BCTQ) severity score and the visual analog scale (VAS) [12, 13].

In this study, we will describe the relationship between CTS and vitamin D deficiency, as well as the effect of vitamin $D$ therapy on decreasing the severity of pain in CTS.

\section{MATERIALS AND METHODS}

This prospective cohort study was performed over a 6-month period, from March to August 2020. Patients who attended the outpatients Department of Cardiovascular Surgery and Middle Euphrates Neurosciences Center, at Al-Sadder Teaching Medical City in Najaf, Iraq, were recruited. CTS was diagnosed according to a patient's history and results of examination and EMG/NCS of the symptomatic limb. All patients $(n=44)$ suffered from hand pain and paresthesia, with or without fatigue. The specific tests performed were Phalen's test and Tinel's test, with a special neurological examination.

Of 44 patients, 32 patients (42 hands) were included in this study. We excluded 12 patients 
who had a positive history of hypothyroidism, liver disease, chronic kidney disease, diabetes mellitus, rheumatoid arthritis, trauma, obesity, normal EMG/NCS, radiculopathy, and a pregnant woman.

The pain assessment was performed using the VAS. Patients were grouped into mild (0-3), moderate (4-7), and severe pain (8-10), using a special line measured from 0-10 and asking patients to select their level of pain. Serum vitamin D levels were checked for all participants, using a venous sample for measuring vitamin $D$ level using a radioimmunoassay method. If a patient's result was less than $20 \mathrm{ng} / \mathrm{ml}$, they were considered vitamin $\mathrm{D}$ deficient.

Each patient received an EMG/NCS using an electromyography device (Deymed Diagnostic device, TruTrace EMG, Czech Republic). The electrodiagnostic studies included the following:

1. A sensory nerve conduction study (NCS) of the median nerve.

2. Sensory nerve action potential (SNAP) assessment.

3. Sensory conduction velocity (SCV) and peakto-peak SNAP were recorded.

4. A motor NCS of the median nerve in the wrist and elbow.

5. Median nerve compound muscle action potential (CMAP) assessment.

6. The distal motor latency (DML) was recorded at the beginning of the CMAP.

7. The baseline to negative peak amplitudes of CMAP and motor conduction velocity (MCV).

A DML of the median nerve of $>4.2 \mathrm{~m} / \mathrm{s}$ and an SCV of the median nerve of $<48 \mathrm{~m} / \mathrm{s}$ were considered abnormal. When there was a normal result in the standard tests, we performed median to ulnar comparison for the fourth finger. If we calculated the fourth finger median and ulnar SNAP peak latency difference to be more than $0.4 \mathrm{~ms}$, we regarded this as abnormal. Regarding the ulnar nerve, motor and sensory conduction studies were measured to exclude its involvement. UInar SNAP was measured by surface electrodes placed over the wrist with a $10 \mathrm{~cm}$ distance from the active stimulating electrode at the fifth digit. Motor NCS was recorded by ulnar nerve stimulation at the wrist, below the elbow $4 \mathrm{~cm}$ down from the medial epicondyle and above the elbow $6 \mathrm{~cm}$ above the medial epicondyle; CMAP was measured by surface electrodes placed over the abductor digiti minimi muscle. Decreased $(<8 \mu \mathrm{V})$ or absent ulnar SNAP and/or reducing MCV over the elbow
$(50 \mathrm{~m} / \mathrm{s})$, or decreased MCV (> $10 \mathrm{~m} / \mathrm{s}$ ) over the elbow as compared with the distal segment and/ or conduction block over the elbow (>20\%) were considered to show the involvement of the ulnar nerve. Patients with involvement of the ulnar nerve were excluded from this study.

According to a modified neurophysiological grading system, patients can be grouped into mild, moderate, and severe NCS findings of CTS. Mild CTS is a median digit-to-wrist segment slowness with normal DML, moderate CTS is defined as a slowing of the median digit-to-wrist segment with abnormal DML, while severe CTS is defined as an absence of median digit-to-wrist segment SNAPS with abnormal DML [14].

Each patient in this study was supplied with oral vitamin $D$ and analgesic drugs and reassessed 3 months later using clinical VAS, EMG/NCS of the previously symptomatic limb, and serum vitamin $D$ levels.

Verbal informed consent was obtained from all patients to be included in this study. The data were analyzed and figures were generated using Microsoft Excel version 2009 and SPSS version 21.0. The data are given as number, mean, standard deviation, and percentage. P-values of $<0.05$ were considered to be statistically significant.

\section{RESULTS}

The study included 32 patients (42 hands), whose median age was $38.5 \pm 7.39$ (range 25 to 50 years). The most common age group was 40 to 49 years (43.75\%) (Figure 1). Most participants (30, $93.75 \%$ ) were female (Figure 2). Most patients' dominant side was their right side $(18,56.25 \%)$, while $10(31.25 \%)$ were bilateral (Figure 3 ). Regarding the EMG/NCS study, patients were grouped into three groups: mild, 16 (50\%); moderate, $12(37.5 \%)$; and severe, 4 (12.5\%) (Figure 4).

The mean vitamin $\mathrm{D}$ level prior to treatment was $12.5 \mathrm{ng} / \mathrm{ml}$ in mild cases, and the mean pain score was 3 . The mean was $10.75 \mathrm{ng} / \mathrm{ml}$ in moderate cases, with a mean pain score of 6 , while in severe cases, the mean was $8 \mathrm{ng} / \mathrm{ml}$ and the mean pain score was 7.5 (Table 1). Following 3 months of treatment, the mean vitamin $D$ level in mild cases was $29 \mathrm{ng} /$ $\mathrm{ml}$ and the mean pain score was 1 . In moderate cases, the mean vitamin D level was $28.5 \mathrm{ng} / \mathrm{L}$, and the mean pain score was 2.5 , while in severe cases, the mean vitamin $D$ level was $28 \mathrm{ng} / \mathrm{L}$ and the mean pain score was 5.5 (Table 2). 


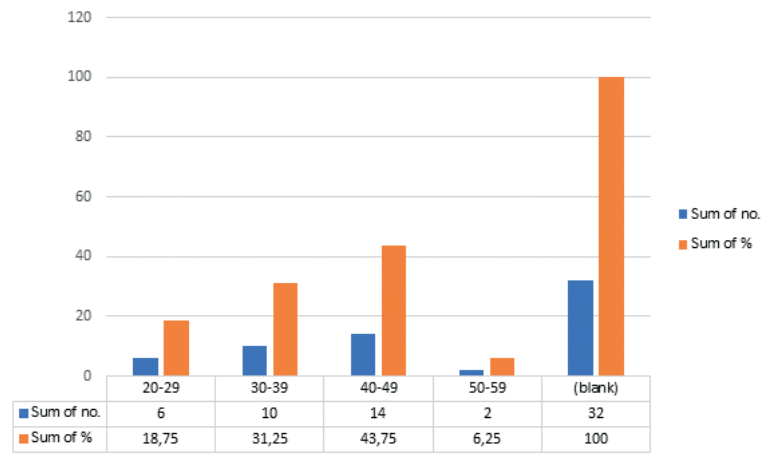

Fig. 1. Patients grouped according to age

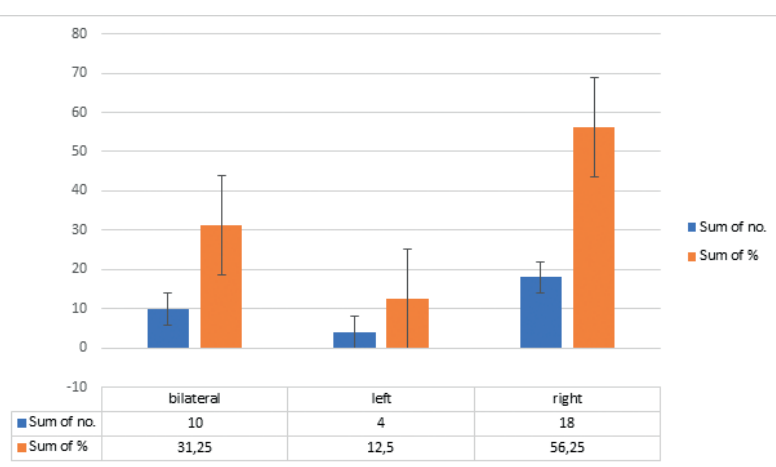

Fig. 3. Sides of the lesion

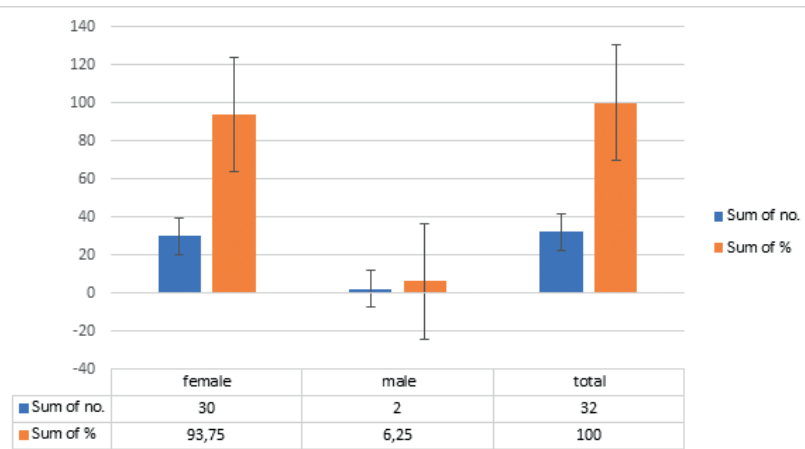

Fig. 2. Sex contribution in CTS patients

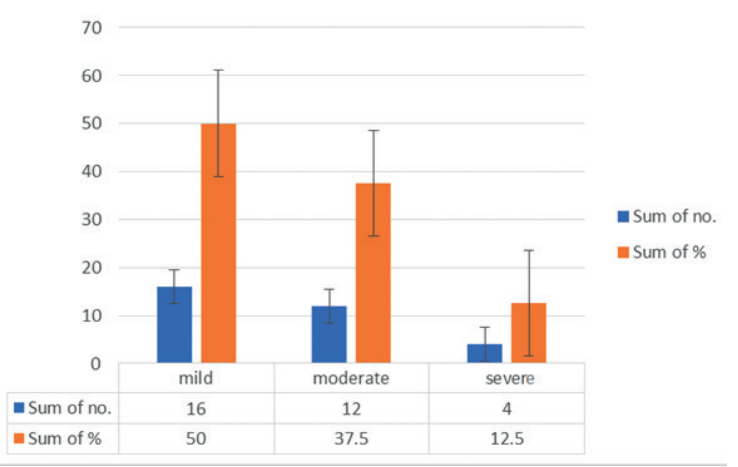

Fig. 4. EMG/NCS study results of all 32 patients

Table 1. Mean level of vitamin $D$ and pain score before treatment

\begin{tabular}{|l|c|c|}
\hline Severity of NCS & $\begin{array}{c}\text { Vitamin D before treatment } \\
\text { (mean } \pm \text { SD) }\end{array}$ & $\begin{array}{c}\text { Pain score before treatment } \\
\text { (mean } \pm \text { SD) }\end{array}$ \\
\hline Mild & $12.5 \pm 3.59$ & $3 \pm 0.45$ \\
\hline Moderate & $10.75 \pm 3.52$ & $6 \pm 0.51$ \\
\hline Severe & 8 & 7.5 \\
\hline
\end{tabular}

Table 2. Mean level of vitamin D and pain score after three months of treatment

\begin{tabular}{|l|c|c|}
\hline Severity of NCS & $\begin{array}{c}\text { Vitamin D after treatment } \\
\text { (mean } \pm \text { SD) }\end{array}$ & $\begin{array}{c}\text { Pain score after treatment } \\
\text { (mean } \pm \text { SD) }\end{array}$ \\
\hline Mild & $29 \pm 6.25$ & $1 \pm 0.62$ \\
\hline Moderate & $28.5 \pm 5.94$ & $2.5 \pm 0.54$ \\
\hline Severe & $28 \pm 5.56$ & $5.5 \pm 0.7$ \\
\hline
\end{tabular}

\section{DISCUSSION}

The prevalence of vitamin $D$ deficiency varies in Iraq, as in many other countries. In Rabar et al.'s study, the prevalence of vitamin $D$ deficiency $(<30$ $\mathrm{mg} / \mathrm{l})$ in Erbil city, Iraq was 78\% [15], while in Babylon city, Iraq, the prevalence was about $50 \%$ [6]. Kimberly et al.'s study showed the overall prevalence to be $41.6 \%$ [5]. Also, in some studies, the incidence of vitamin D deficiency was found to be higher among those with obesity, hyperlipidemia, hypertension, cancer, metabolic diseases, diabetes, and a low level of education $[5,16]$. Vitamin $D$ is important for decreasing inflammation and promoting immune system activity, neuro-muscular action, and health of the skeletal system, so its deficiency can result in chronic pain [17]. 
Our study explored the relationship between vitamin $D$ deficiency and CTS in patients and the effect of 3 months of vitamin $D$ supplements on decreasing the severity of patients' pain. Here, the age group most commonly affected by CTS was 40 to 49 years ( 14 out of 32 patients, $43.75 \%$ ), while the next most-affected population was those aged 30 to 39 years (ten patients, $31.25 \%$ ), with a median age of $38.5 \pm 7.39$. This is different from the study by Shaimaa et al., in which the mean age of patients was $49.2 \pm 10.6$ years [1], and it also differed from the study by Gaurav et al., who reported a mean age of $43.9 \pm 14$ years [9].

In our study, more females $(30,93.75 \%)$ than males were affected, and most of them were housewives. This is similar to the findings of Praveen et al., who reported 39 female and 11 male patients [10]. This increased incidence among females may be due to their staying in the house without exposure to sunlight.

The most common side affected in this study was the dominant right-side (18 patients, $56.25 \%$ ), which may be due to excessive use of the dominant right hand, while it was bilateral in about ten patients (31.25\%). Conversely, Praveen et al. study found 19 out of 50 patients were right-side dominant and 20 out of 50 patients were bilateral [10].

There was a correlation between the level of vitamin $\mathrm{D}$ deficiency and pain score, with more severe vitamin $D$ deficiency resulting in more severe pain scores. This agreed with Shaimaa et al.'s study, where they reported the most severe CTS symptoms were identified in patients with vitamin $\mathrm{D}$ deficiency [1].

\section{CONCLUSION}

In conclusion, there is a relationship between vitamin $D$ deficiency and the severity of carpal tunnel syndrome; clinical symptoms of carpal tunnel syndrome may be decreased by vitamin $D$ therapy.

Acknowledgment: The authors wish to acknowledge Dr. Ahmed Alshewered for his helping.

Conflict of interest: None.

\section{References}

1. Shaimaa M Okasha, Maii A Abdel-latif, Marwa Orabi et al. Evaluation of Vitamin D level in Women with Carpal Tunnel Syndrome. suez canal university medical journal. 2018.20:136-141.

2. Demiryurek BE, Gundogdu AA. The effect of vitamin D level on pain in carpal tunnel syndrome. Sakarya Training and
Research Hospital, Sakarya, Turkey. orthopedic \& traumatology: Surgery \& Research. 2017;103:919-922.

3. Wimalawansa SJ. Non-musculoskeletal benefits of vitamin D. the journal of steroid biochemistry and molecular biology. 2018;175:60-81.

4. Maria Helde-Frankling and Linda Bjorkhem-Bergman. Vitamin D in pain management. Int. J. Mol. Sci. 2017;18(10).

5. Forrest KYZ, Stuhldreher WL. Prevalence and correlates of vitamin $D$ deficiency in US adults. Nutrition Research. 2011;31(1):48-54.

6. Borissova A-M, Shinkov A, Vlahov J, Dakovska L, Todorov $\mathrm{T}$, Svinarov $\mathrm{D}$, et al. Determination of the optimal level of $25(\mathrm{OH}) \mathrm{D}$ in the Bulgarian population. Endocrinologia 2012;17(3):135-42.

7. Hantoosh HA, Mahdi MH, Imran BW et al. Prevalence of Vitamin $D$ deficiency in Iraqi female at reproductive age. Medical journal of Babylon. 2019;16(2):119-122.

8. Singh DP, Rahman SA, Prasad K. Carpal tunnel syndrome: A clinical and electrophysiological appraisal in carpal tunnel syndrome. Journal of medical science and clinical research. 2016;4(11):14004-14009.

9. Kasundra GM, Sood I, Bhargava AN et al. Carpal tunnel syndrome: analyzing efficacy and utility of clinical tests and various diagnostic modalities. J Neurosci Rural Pract. 2015; 6:504-10.

10. Srikanteswara PK, Cheluvalah JD, Agadi JB, et al. The relationship between nerve conduction study and clinical grading of carpal tunnel syndrome. journal of clinical and diagnostic research. 2016;10(7): OC13-OC18.

11. Cunha TAL, Oliveira Filho OM, Ribeiro MB. Phalen test positivation time and it's correlation with electroneuromyography. Acta Ortop Bras. 2020;28(3):114-6.

12. Ollivere BJ, Logan $\mathrm{K}$, Ellahee $\mathrm{N}$ et al. Severity scoring in carpal tunnel syndrome helps predict the value of conservative therapy. J hand surgery Eur. 2009;34(4):511-5.

13. Wewers ME, Lowe NK. A critical review of visual analogue scales in the measurement of clinical phenomena. research in nursing and health. 1990;13:227-236.

14. Padua L, Lomonaco M, Gregore B et al. Neurophysiological classification and sensitivity in 500 carpal tunnel syndrome hands. Acta Neurol Scand. 1997;96:211-217.

15. Rabar M. Abdulrahman, Balen Muhsin Abdul Rahman. Prevalence of vitamin $D$ level in the serum of patients living in Erbil city, Iraq, referred to private clinic laboratory and effect of age and sex on it. Journal of biological research. 2018;91:6916.

16. Parva N R, Tadepalli S, Singh P et al. Prevalence of Vitamin $D$ deficiency and associated risk factors in US population (2011-2012). Cureus. 2018;10(6): e2741.

17. Martin KR, Reid DM. Is there a role for vitamin $D$ in the treatment of chronic pain?. Ther Adv Musculoskel Dis. 2017; 9(6):131-135.

\section{Submitted: 17.06.2021}

\section{Correspondence address:}

Hasanain A. Al-Khalidi, MD, PhD

Najaf, Iraq

Clinical Neurologist

09647733962400

e-mail: ayam.mohammad@yahoo.com 